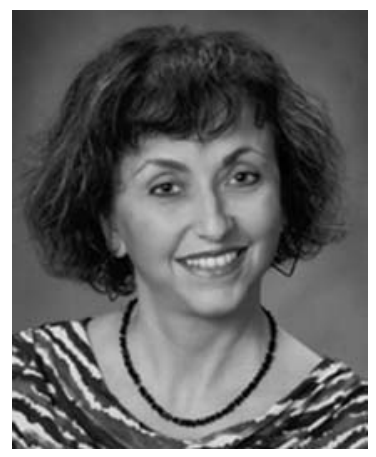

\title{
A Conversation with Dora Angelaki
}

\author{
INTERVIEWER: GARY STIX \\ Senior Editor, Scientific American
}

Dora Angelaki is the Wilhelmina Robertson Professor and Chair of the Department of Neuroscience, Baylor College of Medicine, with a joint appointment in the Departments of Electrical and Computer Engineering and Psychology, Rice University.

Gary Stix: You gave a talk here yesterday at the conference on how neurons that process sensory information can predict how an animal behaves during a simple perceptual task-I think it was a monkey moved in the world? Can you tell us briefly what the field of multisensory integration is attempting to do? Understanding how one sense works is difficult enough, so I assume that trying to integrate information from multiple senses is even more difficult. Are neuroscientists so attracted by this problem because it's a really tough challenge?

Dr. Angelaki: This is only part of the answer. In the laboratory, we like to simplify the enormous task of understanding how the brain works. Traditionally, neuroscience has studied one sensory system at a time. But in the real world, this is not what happens. As we talk now, I look at you, I see your mouth moving and I hear your voice. This experience is multisensory and our brain is optimized to process all these signals simultaneously.

Gary Stix: You don't stop and say I'm about to look at you.

Dr. Angelaki: No, it all happens together and my perception of you, of the world, of what you say, is based on many sensory signals all acting in unison simultaneously. How that happens in the brain, where it happens in the brain, what computations the brain uses - these are the challenges we seek to identify and solve. There are also different layers of understanding: different people have approached this question from the point of view of psychology and psychophysics, physiology, and/or normative theory.

How can the brain abstractly combine multiple sensory signals into a unified experience? How neurons or a network of neurons actually do this is largely unknown. We work at all levels simultaneously. We take advantage of lessons learned from theory and psychophysics, and try to apply them to neurophysiology.

Gary Stix: In your talk, you described experiments in multisensory integration that looked at the integration of visual and vestibular information as a monkey is moved. Vestibular information is information that we use to sense how our bodies are moving and how we can retain our sense of balance. Can you tell us how you went about doing that experiment?

Dr. Angelaki: When we do simple things like riding a bike or driving a car, we use many sensory signals, including input from our vestibular (also known as the balance system), somatosensory, and visual systems. As we move in the world, our retina sees the velocity of things moving relative to us, our accelerometers in the inner ear are activated, and we receive proprioceptive information from our muscles and joints. For example, when we are in a plane, we feel that it is moving even without looking outside the window.

Gary Stix: Or even just walking around.

Dr. Angelaki: Absolutely. But we don't separate the perceptions - oh, this is my vestibular sense, or this is my proprioceptive sense.

Gary Stix: We wouldn't be able to function in the environment.

Dr. Angelaki: Not without the brain being able to put all of these signals together to try to figure out how we move in the world. This is important, not just to perceive the world but to interact with it. Sometimes when we walk on the sidewalk, we turn to look at a shop window. We do this and still maintain our heading. We can talk to our friend, move our head around, and the brain manages to do all of this because of the interaction of many sensory and motor systems.

In the lab, we have set up virtual reality systems. We study human perception, as well as those of animals, macaques and rodents, to try to understand how neurons in the brain combine multiple sources of complementary, but also often redundant, information. In fact, the kind of equipment we use is very similar to what pilots use during training. 
Gary Stix: In a flight simulator?

Dr. Angelaki: It's very much like the flight simulators used for pilot training or those used in amusement parks, in which they accelerate you, as you experience visual stimulation, and you feel like you are moving long distances through space, when you are actually only moving a few centimeters. It is the combination of the stimuli sensed by the ear and body receptors, in combination with the visual stimuli sensed by the retina that gives you the illusion that this is very real. This is what we're doing in the lab. We record brain activity during virtual reality, which allows us to dissociate sensory signals, and thus to understand how we orient and navigate in the world.

Gary Stix: There was one thing that you talked about that I thought was particularly surprising. You looked at the activity of a single neuron and found it could predict what the subject might perceive.

Dr. Angelaki: It is indeed surprising. We knew from other studies that in trained animals, one can find neurons in the brain that are as sensitive as our perception. So if a single cell can do it so well, why do we have so many? In our experiment, rather than recording only from the cortex, we also recorded from the actual receptors from the inner ear, from the vestibular nerve, as well as neurons in the brainstem and cerebellum. We found that even single sensory afferent fibers are as sensitive to motion as our perception. We have 3000 of these neurons carrying information from each motion receptor in the ear to the brain. If one of them is so good, then why do we have so many?

Gary Stix: A single neuron could predict what?

Dr. Angelaki: There are two types of analysis. What I've described so far is how the neuron responses correlate with the stimulus. But, in addition, we record from neurons while the animal performs a task. Then we can also correlate the activity of those neurons with the perceptual choice that the animal makes.

Gary Stix: How the brain decodes the information?

Dr. Angelaki: That's it exactly. The first process is what we call encoding - how the neurons map the sensory world into neural activity. The second process is decoding, which is how the brain takes the activity of all the neurons and generates our perception of the world - critical to orient and be able to initiate an action. We don't know where this happens and any of the details, but that is what we are looking for.

Gary Stix: From that one neuron in the inner ear all the way into the cortex, you could predict what was going to happen?

Dr. Angelaki: Not exactly. It happens only with neurons in the central nervous system. All the neurons are nearly as sensitive as the behavior, but signals about decoding are found only centrally. What is interesting is that neurons with significant correlations with the behavioral choice are not only found in the cortex, but also lower in the brainstem and cerebellum, which are the very early sensory areas that process vestibular signals. You can listen to these neurons and predict what the animal's choice will be. This has many implications about how our brain works and why it doesn't do better. I presented a model, actually several classes of models.

Gary Stix: You're interested in theory and how it might apply to neuroscience and how it might help neuroscience understand the complexities of the brain.

Dr. Angelaki: Absolutely, because I strongly believe that without theory we can never understand how the brain works.

Gary Stix: Some neuroscientists are wedded only to experimentation but you seem to be doing both.

Dr. Angelaki: Yes. For me, it's extremely important to involve theory in all aspects of neuroscience. Not only do I think it's extremely important to use theory to understand how behavior is generated, but I also think that theory is important to connect the different levels of analysis from molecules to the network, to the brain and behavior.

Gary Stix: There is a lot of the emphasis now in neuroscience on gaining this big picture. Do you feel it's necessary to emphasize theory in the projects being set up here and in Europe to try to gain this bigger picture?

Dr. Angelaki: Absolutely. The existing approaches focus primarily on technology development so that we can see as big a piece of the brain at once as possible. That's extremely important and a very valuable goal. Some people make a parallel between neuroscience and astronomy. It was only after the discovery of technically excellent telescopes that we were able to make major advances in our exploration of the cosmos.

This is what is needed in neuroscience. But I don't think astronomy would have been as successful if there hadn't been the appropriate theories able to know what to search for and how to interpret massive amounts of data. Without knowing what to look for and what hypothesis to test, massive data can be overwhelming and not appreciated to their true potential.

Gary Stix: One other thing related to the theory. Turning off a neuron to see whether it causes a deficit in the animal is a standard technique in biology and it's heavily relied upon by neuroscience to prove cause and effect for a particular phenomenon. Yet, you said that, in some cases, that doesn't necessarily tell you that much about the function of a neuron. That seems really surprising.

Dr. Angelaki: That was indeed a provocative statement. I want to emphasize that there are many cases where this approach is extremely helpful, particularly when a causal effect on behavior can be demonstrated. However, for negative results (i.e., those where turning neurons on or off lead to no measurable effects on behavior), we cannot necessarily conclude that these neurons do not participate 
in the behavior. It's a negative result I emphasized because I presented an example of such a situation in which we inactivated a group of neurons and, using theory, we could predict both neural and behavioral results regardless of whether or not these neurons participated in the particular task we studied.
Gary Stix: I imagine that provoked a lot of discussion after your talk.

Dr. Angelaki: A lot, and many students came to talk to me afterwards and some of them disagreed, which is great. This is what science is about. 


\section{$\$_{\text {CSH }}^{\infty}$ Cold Spring Harbor Symposia SYMPOSIA On Quantitative Biology}

\section{A Conversation with Dora Angelaki}

Cold Spring Harb Symp Quant Biol 2014 79: 255-257

Access the most recent version at doi:10.1101/sqb.2014.79.02

\section{License}

Email Alerting Receive free email alerts when new articles cite this article - sign up in Service the box at the top right corner of the article or click here. 